\title{
To explore delirium in liver transplant ICU patients and its management.
}

\author{
Huien-Chun Liao ${ }^{1}$, Shu-Ming Chen ${ }^{2}$, Chin-Tang Tu${ }^{3}$, Ying-Chen Chiu ${ }^{4}$, Ya-Lie Ku ${ }^{5 *}$ (Correspondent)
}

${ }^{1}$ NP, Liver Transplantation Team, Chang Gung Memorial Hospital Kaohsiung Branch, Taiwan, R.O.C

${ }^{2}$ Assistant Professor, College of Nursing, Fooyin University, Taiwan, R.O.C

${ }^{3}$ Professor, Center for Teacher Education, National Kaohsiung Normal University, Taiwan, R.O.C

${ }^{4}$ Head Nurse, Liver Transplantation Team, Chang Gung Memorial Hospital Kaohsiung Branch, Taiwan, R.O.C

${ }^{5}$ Associate Professor, College of Nursing, Fooyin University, Taiwan, R.O.C

\begin{abstract}
Methods: This preliminary study explored, through purposive sampling, patients who were hospitalized in the liver ICU of a medical centre in southern Taiwan (IRB approval number 201601364B0) between November 25, 2016, and July 24, 2017. Before collecting the delirium-related data of the patients, a delirium assessment scale was developed by the principal investigator and one nursing practitioner (NP) in the liver ICU. The content validity of the delirium assessment scale was evaluated by three ICU medical and nursing experts. Additionally, one head nurse and one NP observed, assessed, and recorded the delirious behaviours of nine liver transplant ICU patients during the day and night shifts to ensure the consistency of assessment. After ensuring the content validity and consistent assessment of delirium, the NP formally collected the data of liver transplant ICU patients by using the delirium assessment scale.

Findings: The content validity of the delirium assessment scale, evaluated by three ICU medical professionals and one NP, approached 0.975 . The consistencies of assessment for the Richmond Agitation and Sedation Scale (RASS), pain scores, and delirium assessment scale were evaluated by one head nurse and one NP through assessing the data of 9 liver transplant ICU patients and revealed by ICC of $.984, .997$, and .992 . The preliminary 12 liver transplant ICU delirium patients had the hyperactive RASS scores (0-3) and their pain scores were 3-8 for 1 week. Additionally, most patients exhibited glucose ranging from 120 to $300 \mathrm{mg} / \mathrm{dL}$ and $\mathrm{Hb}$ ranging from 7 to $11 \mathrm{mg} / \mathrm{dL}$. Furthermore, most patients exhibited CRP ranging from 20 to $60 \mathrm{mg} / \mathrm{L}$. The highest frequency of sedative administration was observed in the first week of hospitalization, whereas the pain medication was used for almost 2 weeks. For the 12 liver transplant patients with delirium in the ICU, problems such as constipation and passing urine were managed by using softening agents and diuretics. Moreover, psychiatrists were advised to change the sleeping or sedation medications that were administered to the patients with delirium. Furthermore, the patients underwent restriction, early weaning from ventilator, and peppermint oil wiping to relieve the distention of the abdomen. The use of earplugs and watching TV were also applied to the liver transplanted patients with delirium as alternative interventions to relieve their delirium symptoms.

Conclusion: Delirium persisted for longer in the liver transplant ICU patients than in general ICU patients. Furthermore, higher glucose and infection, and lower $\mathrm{Hb}$ were observed in the 12 liver transplant delirium patients. Sedatives were frequently administered in the first week of hospitalization for managing delirium, while pain medication was administered for almost 2 weeks in the 12 liver transplant ICU delirium patients. In addition to the medications, alternative treatments and interventions were applied to the $\mathbf{1 2}$ liver transplant ICU delirium patients for managing their delirium.
\end{abstract}

Keywords: Delirium, ICU, Liver transplant, Patients.

Accepted on 21 February, 2018

\section{Clinical Relevance}

This preliminary study demonstrates that delirium in liver transplant ICU patients could be prevented, controlled, and managed based on these scientific findings by the strategies designed by the healthcare professionals.

\section{Background}

Intensive Care Units (ICUs), which provide $24 \mathrm{~h}$ service to patients, have a peculiar and closed environment. $\mathrm{Wu}, \mathrm{Gao}$, and Ming [1] observed that patients in ICUs exhibit changes in the levels of neurotransmitters in the brain due to a lack of stimuli from the external environment because they are in a closed space. Consequently, these patients often fail to cooperate 
during treatment, thus leading to delirium. A study indicated that the incidence of delirium in 484 medical and surgical ICU patients at a medical centre in northern Taiwan was 7.44\% [2]; however, the incidence of delirium in 30 conscious clear surgical ICU patients at a medical centre in central Taiwan was $46.7 \%$. Similarly, the incidence of delirium was $50 \%$ in 110 ICU patients from a medical centre in southern Taiwan $[3,4]$. Specifically, Lin et al. [5] reported that $7.8 \%$ of 71 patients who underwent liver surgery developed the postoperative delirium following hepatectomy for the treatment of hepatocellular carcinoma (HCC) at a hospital in central Taiwan. Additionally, Wang et al. [6,7] reported that 58.3\% (21/36) of alcoholic patients and 31.5\% (17/54) of nonalcoholic patients who underwent living donor liver transplantation (LDLT) experienced at least one episode of delirium at a hospital in central Taiwan.

Most ICU patients cannot cooperate with their treatments owing to delirium and cognitive disorders resulting from critical changes in the illness and environment, thus leading to the occurrence of complications, prolong the weaning time from ventilation and the tube insertion rate, the duration of hospitalization, care needs of the patients, medical cost, and an increase in the death $[1,2,4]$. Furthermore, Chen et al. [3] reported that among patients who required mechanical ventilation, the duration of hospitalization, average cost of hospitalization, and death rate were significantly higher for patients with delirium than for those without. Nevertheless, Olson [8] stated that critical care nurses should identify delirium early because it is among the most common problems in ICU patients but is often overlooked or under-recognized. If the health care professionals identify delirium in ICU patients early, they can prevent the subsequent disorientation, confusion, non-cooperation, and unusual or irritable behaviours in the patients. Consequently, the hospitalization duration and death rate of ICU patients with delirium could be reduced, and the function and quality of life of these patients might improve.

\section{Purpose of the Study}

This preliminary study explored delirium in liver transplant ICU patients and its management at a medical centre in southern Taiwan.

\section{Literature Review}

In the literature review focused on delirium-occurrence, related factors, assessment scales, treatment and nursing interventions for the management of delirium in ICU patients.

\section{Occurrence}

Chen et al. [3] reported that, among $82.7 \%$ of the 110 ICU patients in their study, delirium occurred 1 day after ICU admission and persisted for $3.0 \pm 1.8$ days on average and that it occurred most frequently in low-activity patients. For liver ICU patients, Wang et al. [6] explored the 54 delirium patients with alcoholic cirrhosis in ICUs after they had undergone LDLT and reported that delirium occurred $5.9 \pm 3.5$ days after ICU admission and persisted for $4.2 \pm 1.8$ days on average.
Furthermore, Wang et al. [7] explored the 54 patients with nonalcoholic cirrhosis in ICUs who had undergone LDLT. They reported that delirium occurred $8.4 \pm 7.1$ days after ICU admission and persisted for $6.0 \pm 3.0$ days on average.

\section{Related factors}

Arend and Christensen [9] critically reviewed 58 articles concerning ICU-related delirium and identified two categories of risk factors, namely predisposing (advanced age, dementia, transfer from nursing home, sensory impairment, material abuses, elevated urine-creatinine ratio, history of stoke, CHF, epilepsy, depression) and precipitating (acute illness and pharmacology) factors, as well as three categories of contributing factors, namely environmental, iatrogenic, and emotional factors. Additionally, Fu et al. [2] reported that, among the physical and psychological aspects, age, sedation frequency, alarm anxiety, and confusion predicted delirium in 484 ICU patients. Furthermore, delirium in 30 conscious clear surgical ICU patients was significantly related to their fever, the rate of tube insertion, and restriction used [4]. However, Nouwen et al. [10] reported that the findings regarding the relationships between delirium, emotional consequences, and delusional memories were inconsistent after a systematic review of 14 articles. For liver ICU patients, Lin et al. [5] proposed that advanced age and low postoperative haemoglobin $(\mathrm{Hb})$ levels are potential risk factors contributing to the development of postoperative delirium following hepatectomy in patients with HCC at a hospital in central Taiwan. Furthermore, Wang et al. [6] explored the predisposing factors for delirium in 54 patients with alcoholic cirrhosis in ICUs after they had undergone LDLT and identified hepatic encephalopathy as a crucial risk factor for delirium.

\section{Assessment scales}

The authors reviewed literature on assessment scales for patients with delirium and identified the Confusion Assessment Method for the Intensive Care Unit (CAM-ICU) [11] as an effective and rapid assessment tool for nurses to evaluate delirium in patients. Additionally, Chung et al. [12] evaluated the reliability and validity of the Chinese version of the CAMICU by using data from 31 patients at a medical centre ICU in southern Taiwan. They found that its validity (assessed using the prevalence- and bias-adjusted kappa value) was 0.48 ( $\mathrm{p}<$. 01 ) and its McNamara's test value was $p=.72$. Furthermore, when the CAM-ICU was administered by two nursing practitioners (NPs, interviewers), the sensitivity calculated approached $89 \%$; the sensitivity was $96 \%$ when performed by a doctor. Consequently, the CAM-ICU was considered suitable for ICU nurses to detect the delirium easily and rapidly.

Olson [8] reported that the two validated assessment scales for identifying the predisposing factors for delirium in ICU patients were the CAM-ICU and the Intensive Care Delirium Screening Checklist (ICDSC). However, Gusmao-Flores et al. [13] systematically reviewed nine studies that evaluated the CAM-ICU by using the data of 969 patients and four studies that evaluated the ICDSC by using the data of 361 patients, and they reported that the sensitivity and specificity of the CAM- 
Citation: Liao HC, Chen SM, Tu CT, Chiu YC, Ya-Lie K, et al.. To explore delirium in liver transplant ICU patients and its management. $J$ Intensive Crit Care Nurs. 2018;1(1):4-8.

ICU were $80 \%$ and $95.9 \%$, respectively, whereas those of the ICDSC were $74 \%$ was $81.9 \%$, respectively. Additionally, the diagnostic odds ratio of the CAM-ICU was 103.2, and the pooled area under the summary receiver operating characteristic curve (AUC) was 0.97; the diagnostic odds ratio of the ICDSC was 21.5, and its AUC was 0.89. Therefore, the CAM-ICU was superior to the ICDSC as a scale for the assessment of delirium in the patients in the ICU.

\section{Treatment and nursing interventions}

For assessing the effect of pharmacological approach, Arend and Christensen [9] critically reviewed 58 articles related to ICU delirium and identified some drugs that were commonly used in the ICU that were associated with delirium, namely anticholinergic drugs and sedatives. The anticholinergic drugs included atropine, ipratropium bromide, and antibiotics, and the sedatives included clonidine, benzodiazepines, ketamine, morphine, fentanyl, ranitidine, aminophylline, atenolol, corticosteroids, cyclizine, and haloperidol. Additionally, Wu et al. [1] proposed physiological, psychological, and environmental care interventions for ICU patients. The physiological aspect included the data for electrolytes, blood routine, and medication. The psychological aspect included anxiety, worry, fear, hyperactivity (as irritability), and hypoactivity (as apathy). The environmental aspect included quiet, comfort, music, and familiar personal utilities. Additionally, Olson [8] suggested that critical care nurses should apply preventive strategies such as a modified environment to maintain the sleep-wake cycle of ICU patients.

\section{Methods}

This study purposively sampled patients admitted in the liver ICU of a medical centre in southern Taiwan (IRB approval number 201601364B0) between November 25, 2016, and July 24, 2017. Although the CAM-ICU was a more suitable assessment scale than the ICDSC as a delirium assessment scale for patients with delirium in the ICU according to the literature review, this study applied the Richmond Agitation and Sedation Scale (RASS) [14] to assess the delirious behaviours of liver transplant ICU patients. Ely et al. [14] tested the RASS in 38 medical ICU patients and reported that the RASS could effectively identify different levels of delirium and correctly assess fluctuations in patients over time. Table 1 lists Richmond Agitation and Sedation Scale (RASS) [14].

Table 1. Richmond Agitation and Sedation Scale (RASS) [14].

\begin{tabular}{lll}
\hline Score & Term & Description \\
\hline+4 & Combative & Overtly combative or violent; immediate danger to staff \\
\hline+3 & Very agitated & $\begin{array}{l}\text { Pulls on or removes tube(s) or catheter(s) or has } \\
\text { aggressive behaviour toward staff }\end{array}$ \\
\hline+2 & Agitated & $\begin{array}{l}\text { Frequent non-purposeful movement or patient } \\
\text { ventilator disynchrony }\end{array}$ \\
\hline+1 & Restless & $\begin{array}{l}\text { Anxious or apprehensive but movements not } \\
\text { aggressive or vigorous }\end{array}$ \\
\hline
\end{tabular}

\begin{tabular}{|c|c|c|}
\hline 0 & $\begin{array}{l}\text { Alert and } \\
\text { calm }\end{array}$ & Spontaneously pays attention to caregiver \\
\hline-1 & Drowsy & $\begin{array}{l}\text { Not fully alert, but has sustained (more than } 10 \\
\text { seconds) awakening, with eye contact, to voice }\end{array}$ \\
\hline-2 & $\begin{array}{l}\text { Light } \\
\text { sedation }\end{array}$ & $\begin{array}{l}\text { Briefly (less than } 10 \text { seconds) awakens with eye } \\
\text { contact to voice }\end{array}$ \\
\hline-3 & $\begin{array}{l}\text { Moderate } \\
\text { sedation }\end{array}$ & Any movement (but no eye contact) to voice \\
\hline-4 & $\begin{array}{l}\text { Deep } \\
\text { sedation }\end{array}$ & $\begin{array}{l}\text { No response to voice, but any movement to physical } \\
\text { stimulation }\end{array}$ \\
\hline-5 & Unarousable & No response to voice or physical stimulation \\
\hline
\end{tabular}

Before collecting the delirium data, the delirium assessment scale was developed by the principal investigator and one NP in the liver ICU and the content validity of the delirium assessment scale, evaluated by three ICU medical professionals and one NP, approached 0.975. The consistencies of assessment for the Richmond Agitation and Sedation Scale (RASS), pain scores, and delirium assessment scale were evaluated by one head nurse and one NP through assessing the data of 9 liver transplant ICU patients and revealed by ICC of . 984, .997, and .992. Table 2 lists Delirium Assessment Scale of the Patients in the Live ICU Unit.

Table 2. Delirium Assessment Scale of the Patients in the Live ICU Unit.

\section{POD}

Shift

Assessment Items

Diagnosis Delirium+/-

RASS score

Pain score (0-10)

Blood Gas(PH)

Blood Gas (HCO3)

Glucose

$\mathrm{Na}$

$\mathrm{K}$

$\mathrm{Ca}$

$\mathrm{P}$

$\mathrm{Hb}$

WBC

CRP

Bun

$\mathrm{Cr}$

Sedation+/-

Pain medication+/-

Nursing intervention 
Copyright by the College of Nursing, Fooyin University \& Liver Transplantation Team, Kaohsiung Chang Gung Memorial Hospital

\section{Results}

\section{Sample characteristics}

The sample characteristics for the preliminary study of the 12 liver transplant ICU patients with delirium included age, education, job, marriage, drinking habits, diagnoses, number of hospitalizations, and duration of ICU stay. Patients' ages ranged from 43 to 65 years with an average age of 55.7 years. Education ranged from the elementary to college level, with
$50 \%$ of the patients having graduated from high school. Among the patients, 50\% ran businesses, 91.7\% were married, and $50 \%$ had a drinking habit. The number of hospitalizations ranged from one to eight with an average of four hospitalizations. The duration of liver ICU stay ranged from 1 to 26 days with an average duration of 16.5 days. Additionally, the diagnoses of the 12 liver transplant ICU patients with delirium included liver cancer, liver cirrhosis with or without alcoholism, liver cell carcinoma, hepatitis B virus infection, hepatitis $\mathrm{C}$ virus infection, $\mathrm{HCC}$, esophageal varices, and the chronic illness as diabetes mellitus and hyperthyroidism. Table 3 lists the demographics of the 12 patients with delirium.

Table 3. Demographics of 12 Delirium Patients.

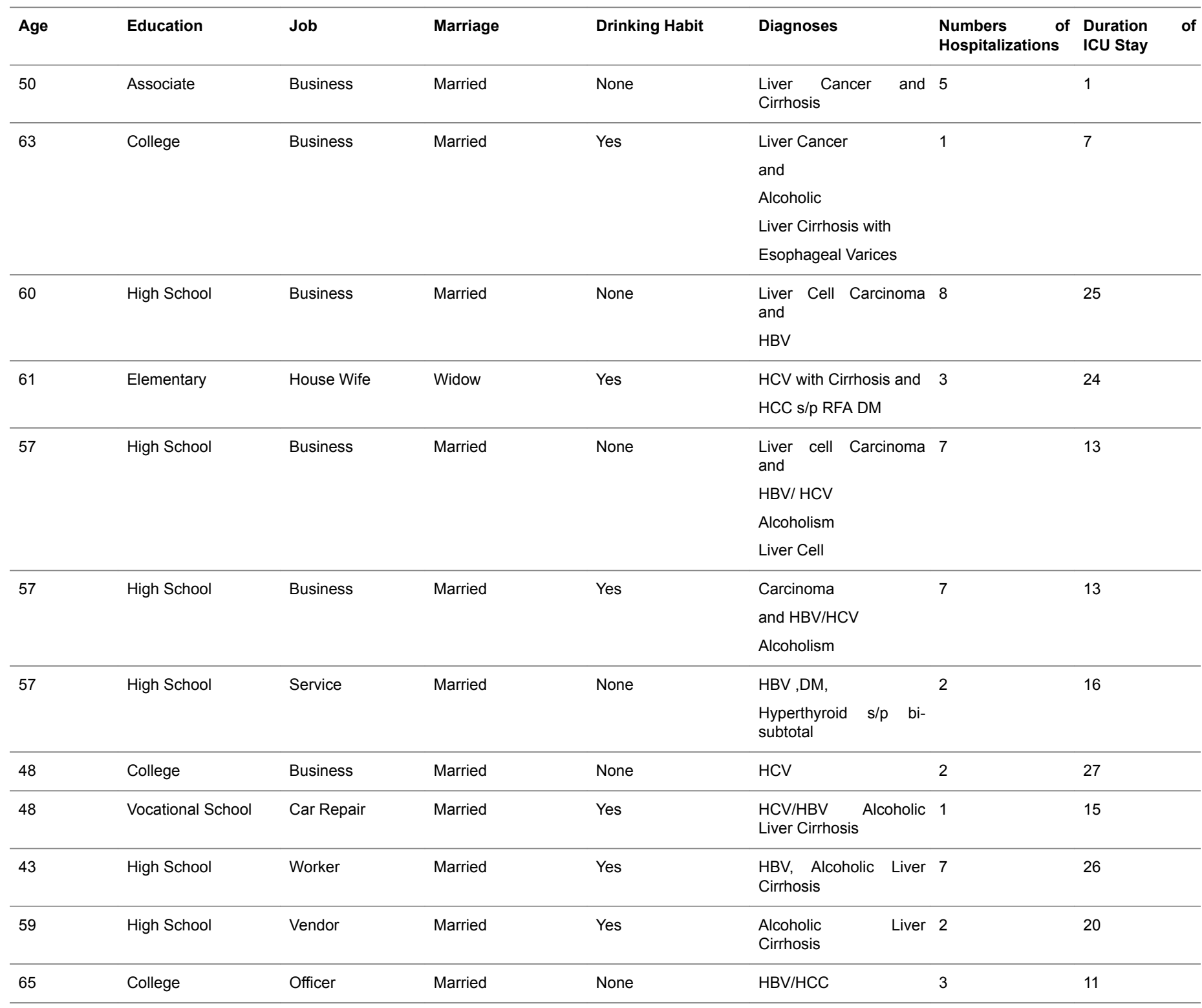

\section{Data in the delirium assessment scale}

The results indicated that the 12 liver transplant ICU patients with delirium in this study had hyperactive RASS scores from 0 to 3 . Their pain scores ranged from 3 to 8 for 1 week and gradually decreased from 8 to 0 over 3 weeks. Additionally, in most of the patients, the glucose level ranged from 120 to 250 $\mathrm{mg} / \mathrm{dL}$ for the first week, which was higher than the standardized level, whereas the $\mathrm{Hb}$ level ranged from 7 to 11 $\mathrm{mg} / \mathrm{dL}$, which was lower than the standardized level. Furthermore, for most of the patients, the CRP level ranged 
Citation: Liao HC, Chen SM, Tu CT, Chiu YC, Ya-Lie K, et al.. To explore delirium in liver transplant ICU patients and its management. $J$

from $20-60 \mathrm{mg} / \mathrm{L}$, which was higher than the standardized level. Figures 1 to 5 depict data of the RASS and pain scores; the levels of glucose, $\mathrm{Hb}$, and CRP.

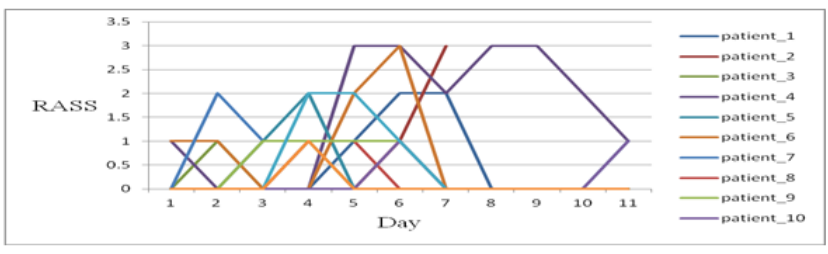

Figure 1. Distribution of RASS scores.

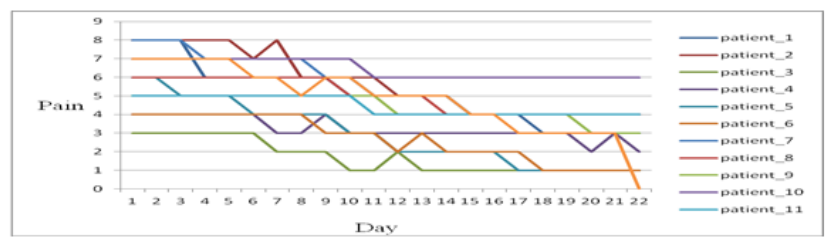

Figure 2. Distribution of Pain scores.

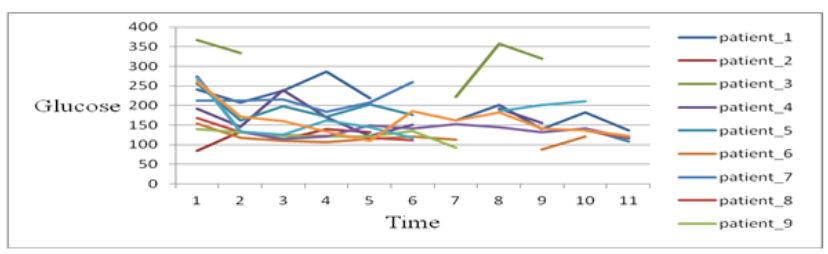

Figure 3. Distribution of Glucose data.

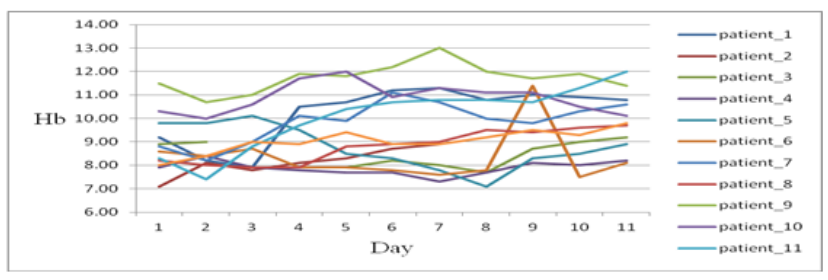

Figure 4. Distribution of $\mathrm{Hb}$ data.

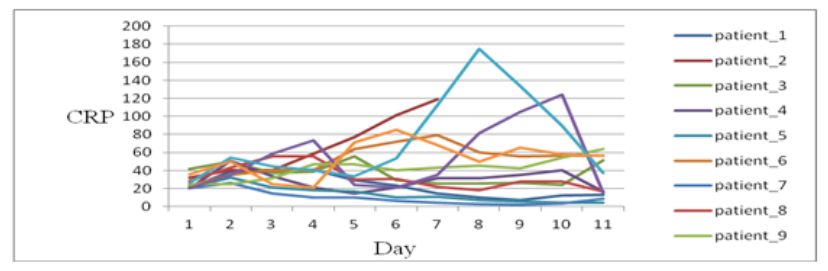

Figure 5. Distribution of CRP data.

In addition to these data, data of the sedation and pain medications were also recorded, as depicted in Figures 6 and 7. The highest frequency of sedation administration was observed in the first week of hospitalization, whereas pain medication was administered for almost 2 weeks to the 12 liver transplant patients with delirium in the ICU. Furthermore, for the 12 liver transplant patients with delirium in the ICU, problems such as constipation and passing urine were managed by using softening agents and diuretics. Moreover, psychiatrists were advised to change the sleeping or sedation medications that were administered to the patients with delirium. Furthermore, the patients underwent restriction, early weaning from ventilator, and peppermint oil wiping to relieve the distention of the abdomen. The use of earplugs and watching TV were also applied to the liver transplanted patients with delirium as alternative interventions to relieve their delirium symptoms.

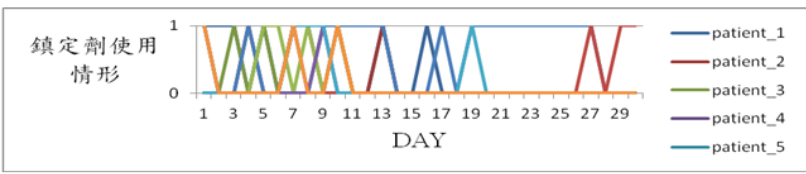

Figure 6. Distribution of Sedation use.

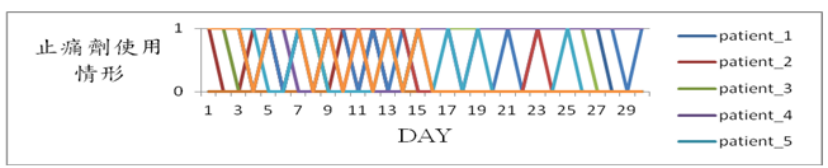

Figure 7. Distribution of pain medication use.

\section{Discussion}

For occurrence of delirium, delirium persisted for longer in the liver ICU than in the general ICU units. Wang et al. [6,7] reported that the durations of ICU stay and hospitalization were significantly longer for both alcoholic and non-alcoholic liver patients with delirium than for patients without delirium; however, these durations were longer for non-alcoholic than for alcoholic liver patients. Among the factors related to delirium in the ICU patients, the findings in this study indicated that infection in the 12 liver transplant ICU patients with delirium had the higher levels of CRP, which was similar to the previous report that listed the physical aspects and predisposing factors for delirium [1,9]. Additionally, the $\mathrm{Hb}$ level was lower than the standardized level in the liver transplant ICU patients in our study, which is similar to low postoperative $\mathrm{Hb}$ levels being a potential risk factor for postoperative delirium following hepatectomy in patients with HCC at a hospital located in central Taiwan [5]. Specially, Wang et al. [6] reported that hepatic encephalopathy was a crucial risk factor for delirium in 54 patients with alcoholic cirrhosis after LDLT in the ICU.

In terms of treatment and interventions, the literature proposed the importance of physiological, psychological, and environmental care interventions for ICU patients [1] For the physiological care, because delirium occurred mainly during the first week, sedation was administered most frequently in the first week of hospitalization to the 12 liver ICU patients with delirium, whereas pain medication was administered for almost 2 weeks. Additionally, other alternative treatments and interventions in this study included managing the discomfort symptoms by using different medications and methods such as restriction, early ventilator removal, peppermint oil wiping, wearing earplugs, and watching TV. However, the interventions used in this study differed from those proposed by Olson [8], namely modifying the environment to maintain the sleep-wake cycle of the ICU patients. 


\section{Conclusion and Suggestions}

In conclusion, delirium persisted for longer in the liver ICU than in the general ICU units. Furthermore, higher and lower levels of infection, glucose and $\mathrm{Hb}$, respectively, than the standardized levels were the major factors observed for the delirium in the 12 liver transplant patients. Instead of treating the major factors, sedation was most frequently administered in the first week of hospitalization to the 12 ICU patients with delirium, whereas pain medication was administered for almost 2 weeks. In addition to the medications, alternative treatments and interventions were applied to the 12 liver transplant ICU patients for relieving their delirium.

Based on the findings of this study, the authors suggested that additional studies are conducted on delirium in the different ICU patients for comparing the durations of delirium, related factors, treatments, and interventions. Additionally, future studies could compare the types of delirium that occur in medical and surgical units as well as in the adult and elderly population for identifying specific delirium treatments and nursing care protocols. Furthermore, future studies could compare the delirium of hospitalized patients with that of those in long-term care facilities for identifying the different related factors for improving the delirium management. Eventually, delirium could be prevented, controlled, and managed by the strategies of the health care professionals based on these scientific findings.

\section{References}

1. Wu SL, Gao FZ, Ming Y. Assessment and nursing care of patients in acute confusion in intensive care units. J Nurs. 2007;54(6):67-72.

2. Fu FH, Shi SF, Chang YC, et al. Acute confusion among intensive care unit patients. Yuan Yuan Nursing. 2007;1(2): 52-63.

3. Chen $\mathrm{MC}$, Chen $\mathrm{CM}$, Ho CC, et al. The exploration of short-term outcomes of delirious patients in the intensive care units. J Healthcare Quality. 2005;9(5):52-63.

4. Chen YL, Sun SG, Huang SW. Delirium among surgical intensive care unit patients. Changhua Nursing. 2012;19(4):33-40.

5. Lin LZ, Hsieh CE, Lin HC, et al. Risk factors for postoperative delirium following hepatectomy in patients with hepatocellular carcinoma. Formosan J Medicine. 2014;18(4):390-6.

6. Wang SH, Hsieh CE, Wang JY, et al. Predisposing factors of delirium for alcoholic liver cirrhosis with living do not liver transplantation in the intensive care units. Taiwan Crit Care Med. 2012;13:178-84.

7. Wang SH, Hsieh CE, Wang JY, et al. Factors associated with delirium in intensive care units of non-alcoholic cirrhotic patients who received living donor liver transplantation. Formosan J Med. 2013;17(6):610-6.

8. Olson T. Delirium in the intensive care unit: role of the critical care nurse in early detection and treatment. Dynamics. 2012;23(4):32-6.

9. Arend E, Christensen M. Delirium in the intensive care unit: a review. Nurs Crit Care. 2009;14(3):145-54.

10. Nouwen MJ, Klijn FAM, Van Den Broek, et al. Emotional consequences of intensive care unit delirium and delusional memories after intensive care unit admission: a systematic review. J Crit Care. 2012;27:199-211.

11. Ely EW, Inouye SK, Bernard GR, et al. Delirium in mechanical ventilated patients. JAMA. 2001;286(21): 2703-10.

12. Chung WL, Lin $\mathrm{CH}$, Hsu W C, et al. Evaluation of the reliability and validity of the Chinese version of the confusion assessment method for the intensive care unit. J Nurs. 2007;54(4):45-52.

13. Gusmao-Flores D, Salluh JIF, Chalhub RA, et al. The confusion assessment method for the intensive care unit (CAM-ICU) and intensive care delirium screening checklist (ICDSC) for the diagnosis of delirium: a systematic review and meta-analysis of clinical studies. Crit Care. 2012;16(R115):1-10.

14. Ely EW, Truman B, Shintani A, et al. Monitoring sedation status over time in ICU patients: reliability and validity of the Richmond Agitation-Sedation Scale (RASS). JAMA. 2003;289(22):2983-91.

\section{*Corresponding author}

Ya-Lie $\mathrm{Ku}$

Associate Professor

Fooyin University, No.151, Chin-Hsueh Rd,

Daliao District, Kaohsiung City 83102,

Taiwan, R.O.C

Tel: 886-7-7811151-5612

E-mail: ns126@fy.edu.tw 